\title{
Analyse des cartes de topographie cornéenne en série après une kératite lamellaire diffuse aiguë unilatérale
}

\author{
Jay Lytle, OD, FAAO \\ Pratique privée, \\ Worthington, Ohio
}

\begin{abstract}
INTRODUCTION
La kératite lamellaire diffuse est une réponse inflammatoire stérile le long de l'interface lamellaire qui peut survenir suite à une chirurgie réfractive cornéenne comme la keratomileusis in situ au laser (LASIK) ou l'extraction d'un lenticule au moyen d'une petite incision (SMILE, small incision lenticule extraction). Elle survient habituellement dans un délai d'un à sept jours après l'intervention, bien qu'on ait rapporté des cas d'apparition tardive ${ }^{1-3}$.
\end{abstract}

La kératite lamellaire diffuse se présente généralement comme de fins dépôts blancs à l'interface qui commencent en périphérie et se propagent de façon diffuse vers le centre de la cornée. Il a été suggéré que la kératite lamellaire diffuse peut représenter une réaction inflammatoire polymorphonucléaire aiguë classique déclenchée par des antigènes ou des toxines introduits dans l'interface cornéenne, sous le volet, pendant la procédure ${ }^{4,5}$. Les sources potentielles comprennent les traumatismes oculaires, les défauts de l'épithélium, le dysfonctionnement des glandes de Meibomius, les endotoxines de la paroi cellulaire bactérienne stérilisées, les résidus d'huile sur l'équipement chirurgical autoclavé (c.-à-d. les microkératomes et les canules), la solution saline équilibrée, les agents environnementaux et les gants chirurgicaux poudrés ${ }^{2,4,5}$. Certains des premiers rapports de kératite lamellaire diffuse ont été publiés en 1998, et l'incidence de cette affection a été estimée à 1 cas sur $500^{2}$. On estime qu'environ $70 \%$ des cas sont unilatéraux ${ }^{2}$.

Au début, les patients sont généralement asymptomatiques. À des stades plus avancés, les symptômes peuvent comprendre une vision floue, une sensation de corps étranger et une sensibilité à la lumière. Dans certains cas, la libération de médiateurs inflammatoires par les amas de globules blancs peut causer une nécrose stromale et une baisse permanente de la meilleure acuité visuelle corrigée en raison de la scarification de la cornée ${ }^{6}$. Les principaux diagnostics différentiels comprennent la kératite stéroïdienne induite par la pression (PISK), la kératopathie centrale toxique (KCT), les débris à l'interface et la kératite bactérienne ${ }^{1,4,5,7-9}$.

Le traitement de la kératite lamellaire diffuse requiert des stéroïdes topiques à tous les stades, et on a observé sa résolution avec une diminution de dose rapide ${ }^{4,5}$. Il est recommandé de soulever les volets et d'irriguer l'interface une fois que l'affection atteint le stade trois, afin d'éviter la conversion au stade quatre ${ }^{7}$. Il a également été démontré que la prise de doxycycline par voie orale et l'administration de citrate de sodium à $10 \%$ sont cliniquement efficaces dans le traitement de la kératite lamellaire diffuse $\mathrm{e}^{10}$. 
Le cas décrit ici présente un résumé quantitatif de la stabilisation topographique qui se produit après la résolution de la kératite lamellaire diffuse, telle qu'observée par examen à la lampe à fente, et permet aux cliniciens d'informer leurs patients des délais liés à la résolution potentielle de la réfraction.

\section{RAPPORT DE CAS}

Un homme de 53 ans s'est présenté au TLC Laser Eye Center (Dublin, OH, É.-U.) avec une kératite lamellaire diffuse aiguë à l'œil droit 11 jours après un LASIK hypermétropique assisté du laser femtoseconde optimisé pour le front d'onde bilatéral.

La réfraction préopératoire manifeste (œil sec) du patient était de $+3,50-0,50 \times 053$ dans l'œil droit et de $+3,75$ 0,50x147 dans l'œil gauche. Les antécédents médicaux du patient étaient positifs pour l'hypertension, l'anxiété et l'hypercholestérolémie, et il a affirmé n'avoir aucun antécédent oculaire pertinent. Il a déclaré avoir pris de l'aténolol, du Prozac et un médicament inconnu à base de statine pour traiter les problèmes systémiques susmentionnés. Il n'avait aucune allergie médicamenteuse connue.

Au cours de l'intervention chirurgicale, on a utilisé une solution saline équilibrée stérile pour l'irrigation intraopératoire normale. Tous les outils chirurgicaux ont été autoclavés à l'aide d'une cassette pour autoclave STATIM et laissés à sécher avant d'être utilisés. Le chirurgien et le personnel portaient des gants chirurgicaux non poudrés. Les volets cornéens du patient ont été créés au moyen d'un laser femtoseconde Alcon FS200. Le diamètre des volets était de $8,8 \mathrm{~mm}$, avec une coupe latérale de 70 degrés et une épaisseur de 120 microns dans chaque œil. Le temps d'aspiration était de 49 secondes pour l'œil droit et de 43 secondes pour l'œil gauche, et la découpe des volets a duré environ huit secondes pour chaque œil. Le traitement par ablation du patient a été effectué au moyen d'un laser excimer Alcon EX500. Le traitement programmé, utilisant le réglage du nomogramme du chirurgien, était de $+3,50-0,25 \times 053$ OD et de $+3,75-0,25$ x 147 OS. L'ablation a duré 13 secondes pour l'œil droit et 14 secondes pour l'œil gauche. L’intervention chirurgicale s'est déroulée sans complications.

Le patient est revenu pour sa première visite postopératoire et a affirmé ne pas avoir de vision floue ou d'inconfort oculaire. Il a déclaré s'administrer de la gatifloxacine quatre fois par jour par instillation dans les deux yeux, de l'acétate de prednisolone $1 \%$ toutes les deux heures dans les deux yeux en période d`éveil, et des larmes artificielles sans agent de conservation toutes les heures dans les deux yeux en période d`éveil, tel que prescrit. Son acuité visuelle de loin non corrigée était de 20/25 à l'œil droit et de 20/25 à l'œil gauche. L'examen à la lampe à fente n'a révélé aucune pathologie de la paupière ou de ses annexes dans l'un ou l'autre des yeux. La conjonctive palpébrale et bulbaire était claire dans les deux yeux, sans signe d'œdème ni d'infection. Les cornées du patient étaient claires, les deux volets cornéens étaient en bonne position, les bords étaient lisses, il n’y avait pas de stries, de brouillard et ou d'opacités à l'interface. La chambre antérieure était profonde et tranquille et il n'y avait aucun signe d'infiltration cellulaire ni de flambée dans l'un ou l'autre des yeux. On a conseillé au patient de continuer ses gouttes oculaires postopératoires selon les directives. En raison de l'horaire du patient, il n'a pas été en mesure de revenir pour sa deuxième visite postopératoire entre les jours 5 et 7, comme recommandé. Il a plutôt pris rendez-vous pour sa deuxième visite de suivi le jour 11. On a conseillé au patient de communiquer immédiatement avec son centre chirurgical s'il remarquait un inconfort oculaire ou une détérioration de sa vision.

La kératite lamellaire diffuse a été observée à la visite postopératoire du jour 11, lorsque l'acuité visuelle non corrigée du patient était de $20 / 50^{-2}$ dans l'œil droit (trou sténopéique $20 / 25^{-1}$ ) et de $20 / 20^{-2}$ dans l'œil gauche. Le patient a signalé une légère vision floue constante et une légère sensibilité à la lumière de l'œil droit. Il a mentionné qu'il dormait avec son ventilateur allumé la nuit et se demandait si la vision floue pouvait avoir été causée par la sécheresse oculaire. En répondant à d'autres questions, le patient a signalé que l'asymétrie de son acuité visuelle et sa sensibilité accrue à la lumière avaient commencé six jours plus tôt (cinq jours après l'intervention chirurgicale). Toutefois, il n'avait pas l'impression que ses symptômes étaient suffisamment importants pour qu'il doive communiquer avec son centre chirurgical. La réfraction manifeste du patient était de $+1,00-0,50 \times 146\left(20 / 30^{+1}\right)$ dans l'œil droit et de $+0,25-0,25 \times 168\left(20 / 20^{-1}\right)$ dans l'œil gauche. Il utilisait de l'acétate de prednisolone $1 \%$ quatre fois par jour dans les deux yeux et sa cornée droite montrait des microstries centrales, une brume stromale diffuse de grade 2+ au niveau de l'interface du volet avec confluence centrale légère et des opacités blanches fines de grade 2+ dispersées le long de l'interface du volet. Le bord du volet était lisse et ne présentait aucun signe d'infiltration épithéliale. La cornée gauche du patient était claire, le volet était en bonne position, sans stries, sans brouillard et sans opacité à l'interface. La fluorescéine de sodium n'a révélé aucun défaut de l'épithélium, et 
aucune hyperémie conjonctivale n'était présente dans aucun des deux yeux. La pression intraoculaire du patient, mesurée à l'aide de la tonométrie d'applanation Tonopen sur la cornée centrale, était de $13 \mathrm{~mm} \mathrm{Hg}$ dans l'œil droit et de $12 \mathrm{~mm} \mathrm{Hg}$ dans l'œil gauche, mesurée à $10 \mathrm{~h} \mathrm{20}$. On a diagnostiqué chez le patient une kératite lamellaire diffuse dans l'œil droit et un traitement à l'acétate de prednisolone à $1 \%$ en suspension ophtalmique (une goutte par heure, en période d'éveil) a été initié à l'œil droit.

La kératite lamellaire diffuse s'est améliorée grâce à une réduction progressive des stéroïdes (toutes les heures pendant une semaine, toutes les deux heures pendant une semaine, quatre fois par jour pendant une semaine, une fois par jour pendant une semaine) et a atteint sa résolution finale au jour 32 de la visite postopératoire (21 jours après le diagnostic initial). Une semaine plus tard (jour 39), le patient a affirmé ne plus avoir de symptôme de vision floue ou de sensibilité à la lumière. À chaque visite postopératoire, on a mesuré les pressions intraoculaires centrales et périphériques du patient pour exclure le PISK, et on a constaté qu'elles se situaient dans la plage normale. Une fois l'affection résolue à l'examen à la lampe à fente, le patient a été vu régulièrement pour faire le suivi de son erreur de réfraction et sa tomographie au Pentacam (Oculus, Wetzlar, Allemagne). Au début, les cartes de courbure axiale/sagittale de l'œil droit montraient des zones locales de forte inclinaison et un aplatissement adjacents à la confluence centrale de la kératite lamellaire diffuse. L'œil gauche présentait une ablation hyperopique relativement uniforme après LASIK. Le jour 88 , le patient est demeuré asymptomatique et ses deux yeux ont montré une ablation hyperopique relativement uniforme après LASIK, avec seulement quelques zones d'aplatissement résiduel au centre de l'œil droit. L'acuité visuelle de loin non corrigée finale du patient était de $20 / 25^{+1}$ dans l'œil droit et de $20 / 15^{-2}$ dans l'œil gauche, avec une réfraction manifeste de $+0,50$ sphère $(20 / 20)$ et de $+0,50-0,50 \times 162(20 / 15)$, respectivement.

Figure 1: Stabilisation topographique

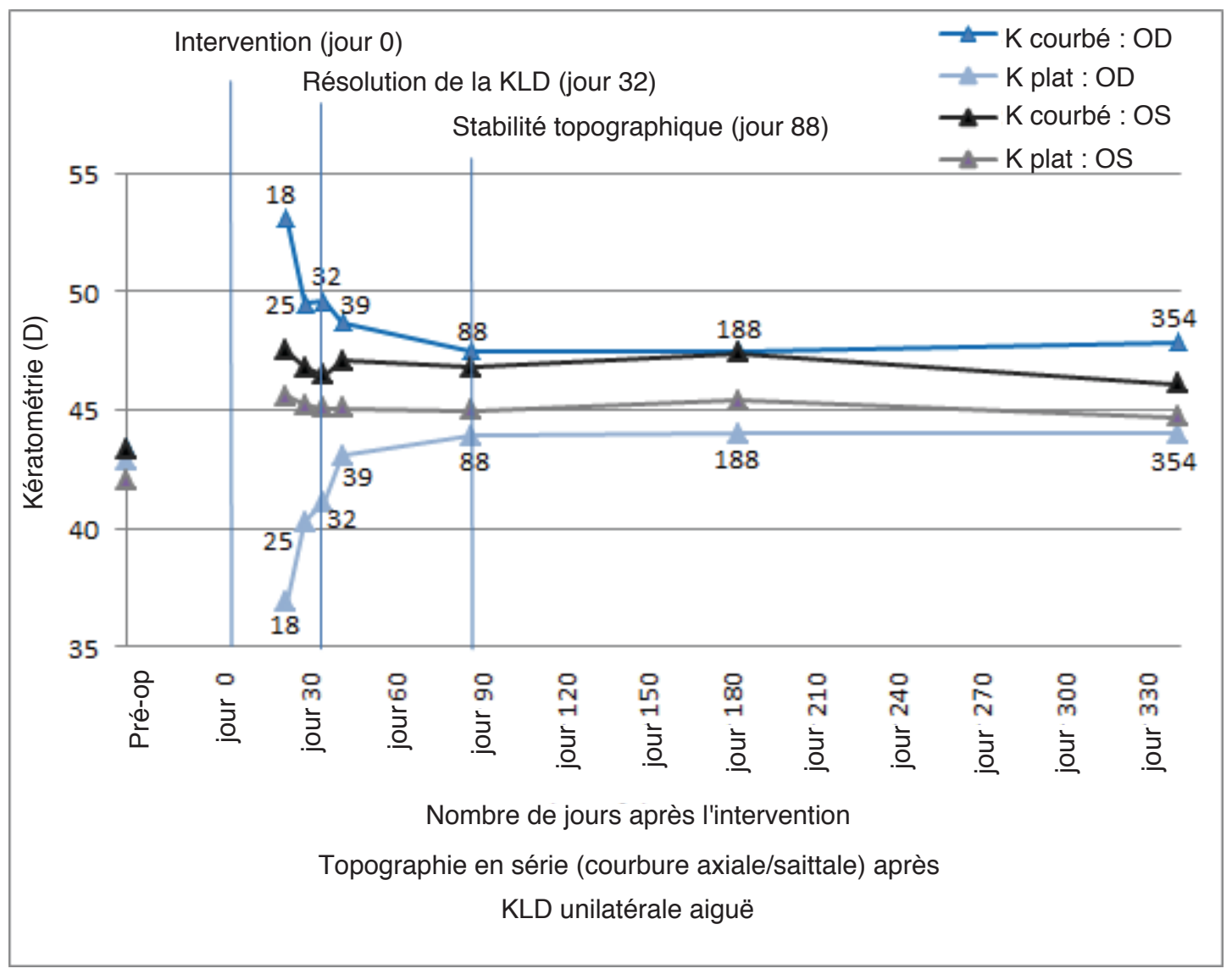


TABLEAU 1: Cartes de courbure axiale/sagittale (Pentacam), cartes de pachymétrie (Pentacam) et résultats cliniques connexes

\begin{tabular}{|c|c|c|c|c|}
\hline & & Eil droit & & Eil gauche \\
\hline & Résultats de l'examen & Axiale / sagittale & Pachymétrie & Axiale / sagittale \\
\hline Examen & Pré-op & & & \\
\hline KLD & S. O. & & & \\
\hline AVNC & $20 / 200$ & & & \\
\hline $\mathrm{RM}$ & $+3,50-050 \times 05320 / 20$ & & & \\
\hline K cambré/K plat & $43,4 \mathrm{D} / 42,9 \mathrm{D}$ & & & \\
\hline Point le plus mince & 531 microns & & & \\
\hline Examen & Jour 11 & & & \\
\hline KLD & Grade 2+ & & & \\
\hline AVNC & $20 / 50^{-2}$ & & & \\
\hline $\mathrm{RM}$ & $+1,00-0,50 \times 146(20 / 30)$ & & & \\
\hline K courbé/K plat & Aucune donnée & & & \\
\hline Point le plus mince & Aucune donnée & & & \\
\hline Examen & Jour 13 & & & \\
\hline KLD & Grade 2 & & & \\
\hline AVNC & $20 / 30^{-2}$ & & & \\
\hline $\mathrm{RM}$ & $+1,00-1,25 \times 151(20 / 30)$ & & & \\
\hline $\mathrm{K}$ courbé/K plat & Aucune donnée & & & \\
\hline Point le plus mince & Aucune donnée & & & \\
\hline Examen & Jour 18 & & & \\
\hline KLD & Grade 1+ & & & \\
\hline AVNC & $20 / 40^{-2}$ & & & \\
\hline $\mathrm{RM}$ & $+1,50-1,75 \times 136(20 / 25)$ & & & \\
\hline $\mathrm{K}$ courbé/K plat & $53,1 \mathrm{D} / 36,9 \mathrm{D}$ & & & \\
\hline Point le plus mince & 447 microns & & & \\
\hline Examen & Jour 25 & & & \\
\hline KLD & Grade 1 & & & \\
\hline AVNC & $20 / 70$ & & & \\
\hline $\mathrm{RM}$ & $+1,25-1,00 \times 146(20 / 25)$ & & & \\
\hline $\mathrm{K}$ courbé/K plat & $49,5 \mathrm{D} / 40,3 \mathrm{D}$ & & & \\
\hline Point le plus mince & 470 microns & & & \\
\hline Examen & Jour 32 & 8 & w. & \\
\hline KLD & Aucune (résolue) & & & \\
\hline AVNC & $20 / 40-2$ & & $\lim$ & \\
\hline $\mathrm{RM}$ & $+1,00-1,00 \times 144(20 / 25)$ & & & \\
\hline $\mathrm{K}$ courbé/K plat & $49,6 \mathrm{D} / 41,1 \mathrm{D}$ & & $p$ & \\
\hline Point le plus mince & 487 microns & & & \\
\hline
\end{tabular}




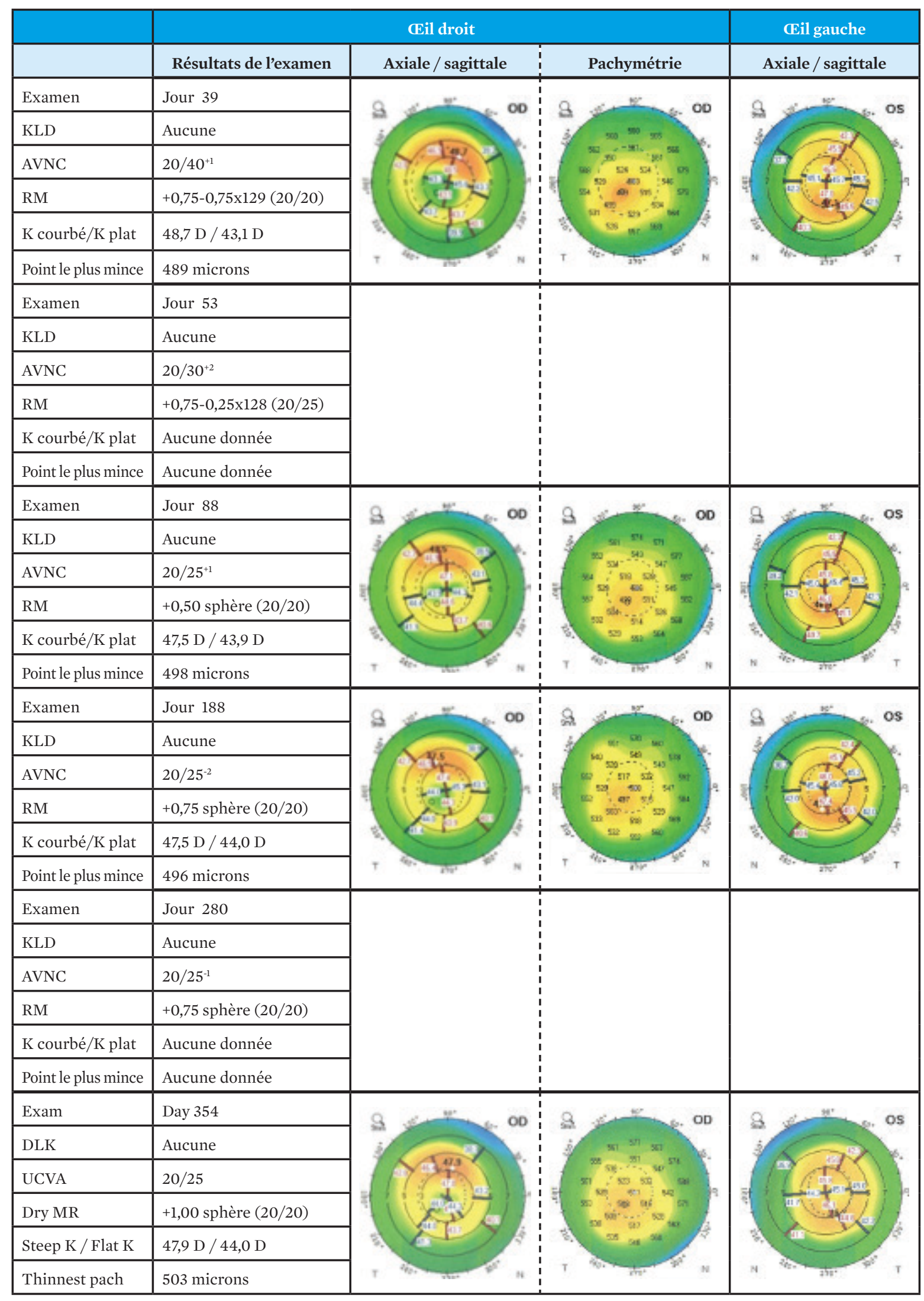




\section{DISCUSSION}

À partir du moment où la kératite lamellaire diffuse du patient s'est résolue le 32e jour, la disparité entre les points les plus courbés et les points les plus plats de sa kératométrie s'est atténuée et l'erreur de réfraction du patient est devenue plus sphérique. Au jour 88, le patient est passé d'une dioptrie d'astigmatisme oblique à une réfraction manifeste complètement sphérique, et d'une disparité de 8,5 dioptries sur ses lectures de kératométrie forte et plate (49,6-41,1) à une disparité de 3,6 dioptries. (47,5-43,9). Au cours des trois autres visites postopératoires du patient (jour 188, jour 280, jour 354), aucun changement significatif de topographie n'a été observé.

Les cartes de pachymétrie Pentacam du patient ont révélé un amincissement cornéen adjacent à la kératite lamellaire diffuse avec des points locaux les plus minces de 531 microns (ligne de base pré-opératoire), 447 microns (jour 18), 470 microns (jour 25), 487 microns (jour 32), 489 microns (jour 39), 498 microns (jour 88 ), 496 microns (jour 188), et 503 microns (jour 354). Comme on l'a noté avec la topographie axiale/sagittale, les résultats de pachymétrie se sont stabilisés au jour 88 .

Par conséquent, il semble que tous les changements cliniquement significatifs associés à la kératite lamellaire diffuse de ce patient se soient produits dans les trois premiers mois suivant le diagnostic initial. La résolution des signes et symptômes cliniques du patient a été observée au cours du premier mois, tandis que la résolution topographique et réfractive a été observée au troisième mois. Des rapports de cas similaires chez des patients atteints de kératite lamellaire diffuse de stade quatre ont signalé une résolution de la topographie et de l'erreur de réfraction après 6 à 12 mois. $^{10,11}$.

Selon la présentation du patient dans cette étude de cas, la gravité de la kératite lamellaire diffuse lors de la présentation initiale était probablement entre les stades deux et trois. La réponse au traitement prescrit a évité d'avoir à effectuer le soulèvement des volets et l'irrigation. La régression hyperopique dans l'œil droit du jour 88 (+0,50 sphère) au jour 354 (+1,00 sphère) peut avoir été causée par la régression hyperopique post-LASIK $(+3,50-0,25 \times 053$ traitée), et n'était pas nécessairement associée à la kératite lamellaire diffuse.

\section{CONCLUSION}

Cette étude de cas montre que la coalescence des globules blancs le long d'une interface de volet peut causer des changements de kératométrie locaux, provoquant souvent une hyperopie et un astigmatisme irrégulier. Ces changements associés à la kératite lamellaire diffuse montrent que le processus de résolution se poursuit même après la disparition des signes cliniques. Dans ce cas, on a observé une stabilité réfractive deux mois après la disparition de la kératite lamellaire diffuse à l'examen à la lampe à fente.

\section{RÉFÉRENCES}

1. Bowling, B. Kanski’s Clinical Ophthalmology: A Systematic Approach, $8^{\text {th }}$ ed. Amsterdam: Elsevier, 2016.

2. Probst LE. Complex Cases with LASIK: Advanced Techniques and Complication Management. Thorofare, NJ: SLACK Inc., 2000

3. Morales P, Camos-Carreras A, Spencer F, et al. Late-onset diffuse lamellar keratitis 15 years after laser in situ keratomileusis. Can J Ophthalmol 2017;52:3, 91-3.

4. Probst LE, Tsai JH. Ophthalmology Clinical and Surgical Principles. Thorofare, NJ: SLACK Inc., 2012.

5. Gimbel HV, Penno EA. LASIK Complications: Prevention and Management. Thorofare, NJ: SLACK Inc., 1999.

6. Boyd BF. LASIK and Beyond LASIK: Wavefront Analysis and Customized Ablation. $1^{\text {st }}$ ed. El Dorado, Panama: Highlights of Ophthalmology International, 2001.

7. Randleman JB, Shah RD. LASIK interface complications: etiology, management, \& outcomes. J Refract Surg 2012;28:575-86.

8. Davidson RS, Brandt JD, Mannis MJ. Intraocular pressure-induced interlamellar keratitis after LASIK surgery. J Glaucoma 2003;12:23-6

9. Sonmez B, Maloney RK. Central toxic keratopathy: description of a syndrome in laser refractive surgery. Am J Ophthalmol 2007;143:420-7.

10. Balestrazzi A, Balestrazzi A, Giannico M, I, et al. Diagnosis, clinica trend, and treatment of diffuse lamellar keratitis after femtosecond laser-assisted in situ keratomileusis: a case report. Ophthalmology 2018;9:457-64.

11. Michieletto P, Balestrazzi A, Balestrazzi A, et al. Stage 4 diffuse lamellar keratitis after laser in situ keratomileusis Clinical, topographical, and pachymetry resolution 5 years later. J Cataract Refract Surg 2006;32(2):353-6. 